\title{
Fast Radar Power Amplifier Optimization for Bandwidth, Efficiency, and Spectral Confinement Using the Smith Tube
}

\author{
Matthew Fellows ${ }^{1}$, Jennifer Barlow ${ }^{1}$, Matthew Flachsbart ${ }^{1}$, Joseph Barkate ${ }^{1}$, Charles Baylis ${ }^{1}$, Lawrence Cohen ${ }^{2}$, \\ and Robert J. Marks II ${ }^{1}$ \\ ${ }^{1}$ Wireless and Microwave Circuits and Systems Program \\ Department of Electrical and Computer Engineering \\ Baylor University \\ Waco, TX, USA \\ ${ }^{2}$ U.S. Naval Research Laboratory \\ Radar Division \\ Washington, DC, USA
}

\begin{abstract}
The Smith Tube, a three-dimensional cylindrical extension of the Smith Chart, provides a mechanism to simultaneously optimize power amplifier circuit and waveform parameters for radar transmitters. A direct, vector-based search algorithm is presented to find the optimum combination of bandwidth and load impedance for a power amplifier to maximize the waveform bandwidth while meeting requirements on the power-added efficiency and the adjacent-channel power ratio. For this optimization, waveform bandwidth is represented on the vertical axis of the Smith Tube, with the complex load reflection coefficient represented in the horizontal plane. Excellent correspondence is obtained in measurement testing of the algorithm from multiple starting combinations of load reflection coefficient and bandwidth. The results of this work are expected to be useful in real-time optimization of power amplifiers for radar and communications. The algorithm is expected to be useful in optimizing the range resolution of radar transmissions.
\end{abstract}

\section{INTRODUCTION}

The radio spectrum is under intense demand from multiple wireless applications as well as radar transmissions. Future systems will need to be reconfigurable to facilitate dynamic spectrum access. The idea of dynamic spectrum allocation will mean that (1) devices will be required to change operating frequencies in real time and (2) the spectral masks placed on devices will vary dynamically based on the spectrum occupancy in surrounding areas. Future radar power amplifiers may need to reconfigure in real-time to change the spectral properties of their output while optimizing performance parameters such as range resolution and power efficiency.

We recently demonstrated the optimization of poweramplifier load impedance to maximize the power-added efficiency (PAE) while keeping the adjacent-channel power ratio (ACPR) below a pre-specified maximum. A vectorbased triangulation algorithm was designed to perform this operation in the well-known two-dimensional Smith Chart [1]. In another recent paper, we introduced the Smith Tube as a three-dimensional, cylindrical extension of the Smith Chart that is useful for joint design of the circuit and the waveform bandwidth. This paper demonstrates a design based upon load-pull measurement data taken for waveforms of different bandwidths [2]. The Smith Tube is shown in Fig. 1. In the present paper, we demonstrate an intelligent, fast algorithm to find the combination of load reflection coefficient and waveform providing the highest bandwidth while meeting requirements on PAE and ACPR. A previously submitted journal manuscript (presently under review) from our group provides information on a two-step algorithm [3]; the present paper provides a single-step, direct approach to seek the optimum.

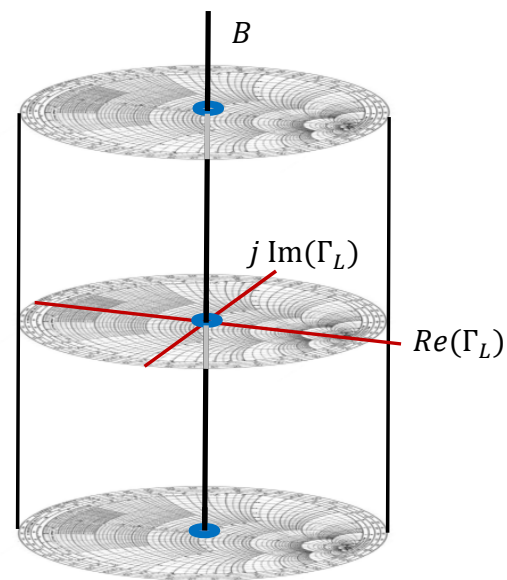

Fig. 1. The Smith tube. The vertical axis represents the bandwidth of the input chirp waveform, while the horizontal cross section of the tube is a conventional Smith chart [2].

The fact that PAE and ACPR are significant functions of the load impedance is well-documented in the literature [4, 5]. The ACPR is based on the third- and fifth-order intermodulation nonlinearities of the device [6], and design compromises for third-order intermodulation and output 
power have been considered by Sechi [7] using a graphical technique based on pre-measured data. Intelligent algorithms have been demonstrated for faster impedance tuning in antennas $[8,9]$, and approaches shown in the literature for fast impedance tuning include fuzzy control [10], neural networks [11], and least-squares optimization [12]. The idea of applying intelligent impedance tuning to high-efficiency transmitters is suggested by Sun et al. [13]. Kingsley and Guerci demonstrate practical implementation of an adaptive amplifier module that can adjust to optimize for different requirements, such as PAE, third-order intermodulation reduction, and output power [14].

Along with optimizing the amplifier load impedance, our algorithm involves optimization of the waveform bandwidth, which has profound significance for radar transmitters. A significant body of literature exists related to waveform optimization for radar. Discussions of waveform optimization include the design of spectrally confined waveforms using variable-modulus techniques [15], constantmodulus techniques (for maximizing power efficiency) such as continuous-phase modulation $[16,17]$ and piecewise linear chirp optimization $[18,19]$, optimization for a target ambiguity function $[20,21,22,23]$, and transmitter-in-theloop optimization [24]. The idea of real-time transmitter reconfiguration, including both real-time waveform and circuit optimization, is particularly becoming attractive for emerging radar protocols. Adaptive radar systems have been proposed to adjust their designs in real-time for changing detection requirements, operating frequencies, and spectrum requirements. Cognitive radar systems learn from and respond to their environments $[25,26]$. While a variety of useful waveform optimization techniques are discussed in the literature, our approach differs from the aforementioned papers in that it includes the optimization of both waveform and circuit parameters.

In Section II, the algorithm's operation is described. Section III describes the results of measurement testing of the algorithm using different combinations of user-defined search parameters. Section IV provides some conclusions regarding the algorithm and its potential applications.

\section{ThreE-Dimensional Vector SEARCH Algorithm}

Figure 2 shows a conceptual drawing of the optimization problem in the Smith Tube. It is desired to find the combination of bandwidth $B$ and load reflection coefficient $\Gamma_{L}$ that allows the largest bandwidth while meeting predefined constraints on PAE and ACPR. Figure 2 shows constant PAE and ACPR surfaces for the PAE and ACPR limits. The power-added efficiency is given by the following equation:

$$
P A E=\frac{P_{o u t, R F}-P_{i n, R F}}{P_{D C}} \times 100 \%
$$

The PAE is dependent upon measurement of the RF power, which is performed with a broadband power sensor. As such, the PAE is expected to be only slightly dependent on dent of bandwidth. Thus the limiting PAE surface is nearly cylindrical, as shown in Fig. 2. On the other hand, the ACPR is expected to grow with increasing bandwidth, causing fewer values of $\Gamma_{L}$ to meet requirements for ACPR as the bandwidth $B$ is increased. As such, the surface containing combinations of $\left(\Gamma_{L}, B\right)$ producing acceptable ACPR is expected to narrow as $B$ is increased, creating a cone-shaped surface, as shown in Fig. 2. The desired solution is the highest intersection point of the PAE and ACPR surfaces, as shown.

The user provides several search parameters to facilitate the search: the starting value for $\Gamma_{L}$, upper and lower limits of the search range for bandwidth $B$, PAE and ACPR limitations, the $\Gamma_{L}$ "neighboring point" resolution distance $D_{n}$, the search distance $D_{s}$, and the bandwidth resolution parameter $B_{\text {stop }}$. The bandwidth range entered by the user is normalized to set the minimum bandwidth to -1 and the maximum bandwidth to 1 . This allows the vertical range of the Smith Tube (-1 to 1$)$ to coincide with the Smith Chart $\Gamma_{L}$ dimension for search consistency and scalability purposes.

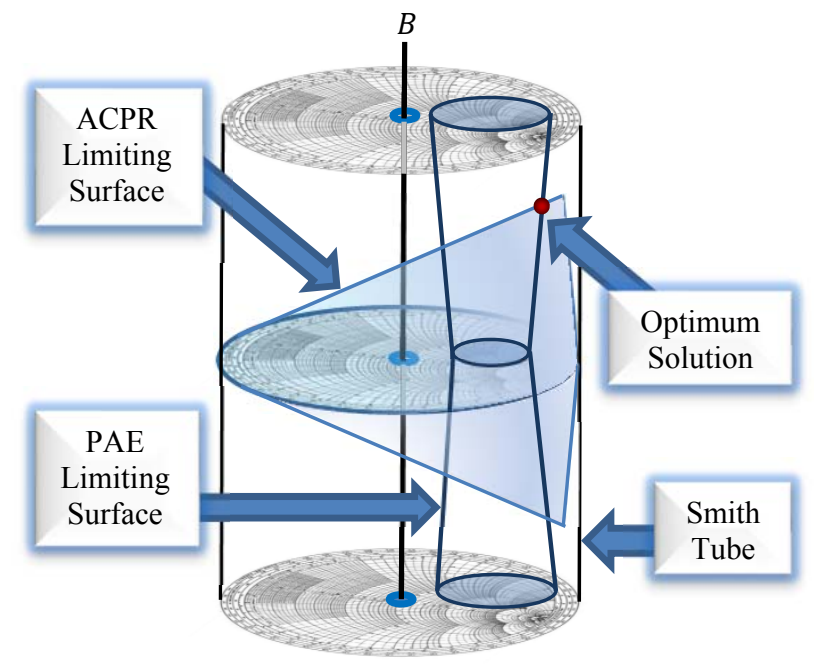

Fig. 2. Conceptual drawing of the Smith tube containing example surfaces for the limiting PAE and ACPR values. The optimum point is selected as the highest point of intersection (representing the largest bandwidth) between the surfaces representing the limiting $\mathrm{PAE}$ and ACPR values [2].

The search adds a third dimension to the two-dimensional "triangulation" approach of [1] for the inclusion of bandwidth $B$ as an input parameter. The step sizes and directions from one search candidate to the next are found by taking measurements to estimate the gradient at the candidate point. Figure 3 provides a conceptual visualization of these gradient evaluation measurements. The two-dimensional gradients of PAE and ACPR are evaluated in the Smith Chart plane by using the two neighboring points in the horizontal plane. A point is measured above the candidate in the vertical $B$ direction to assess the change in ACPR resulting from a small change in waveform bandwidth. The unit vector $\hat{p}$ is in the direction of PAE steepest ascent in the Smith Chart plane and the unit vector $\hat{a}$ is in the direction of ACPR steepest descent 
in the Smith Chart plane. The unit vector $\hat{B}$ is pointed upward.

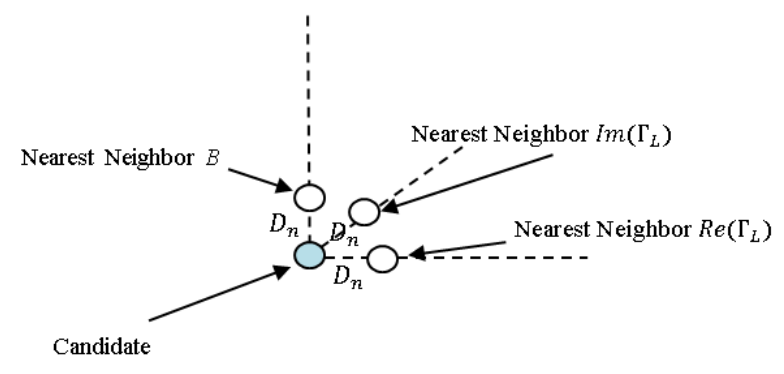

Fig. 3. Measurements for PAE and ACPR variation in the three dimensions

The neighboring-point measurements are used to construct search vectors, allowing the location in the Smith Tube of the next candidate to be identified. In the case where the present candidate possesses acceptable PAE, the vector from the present candidate to the next candidate is given by the search vector

$$
\bar{v}=\hat{p} D_{p}+\hat{b} D_{b}+\hat{B} D_{B}
$$

as shown in Fig. 4(a). Fig. 4(b) shows the search vector in the case where the PAE value at the present candidate is not in the acceptable region:

$$
\bar{v}=\hat{a} D_{p}+\hat{b} D_{b}+\hat{B} D_{B} .
$$

The components of these vectors are given as follows:

$$
\begin{aligned}
D_{p} & =\frac{D_{s}}{2} \frac{\left|P A E_{\text {cand }}-P A E_{\text {target }}\right|}{\left|P A E_{\text {worst }}-P A E_{\text {target }}\right|} \\
D_{b} & =\frac{D_{s}}{2} \frac{\left|\theta_{\text {cand }}-\theta_{\text {target }}\right|}{\theta_{\text {target }}} \\
D_{B} & =D_{n} \frac{A C P R_{\text {target }}-A C P R_{\text {cand }}}{A C P R_{\text {neb,up }}-A C P R_{\text {cand }}}
\end{aligned}
$$

In these expressions, $\theta$ is the angle between $\hat{a}$ and $\hat{b}$ (or equivalently between $\hat{p}$ and $\hat{b}$ ). $P A E_{\text {cand }}, \theta_{\text {cand }}$, and $A C P R_{\text {cand }}$ are the measured PAE, bisector angle, and ACPR measured values, respectively, at the present candidate point. $P A E_{\text {target }}, \theta_{\text {target }}$, and $A C P R_{\text {target }}$ are the goal (limiting) values of PAE, bisector angle, and ACPR. $A C P R_{n e b, u p}$ is the ACPR value measured at the neighboring point in the upward direction.

Equation (4) provides the distance to be traveled in the PAE direction (or the ACPR direction in the case where the present candidate is in the PAE acceptable region) by calculating the relative distance between the value of PAE at the candidate and the target limiting value to the distance between the worst value measured and the target. This is, in some sense, an estimator of the distance remaining to be traveled divided by the total distance traveled from a worstcase starting point. As the search converges toward $P A E_{\text {target }}$, the value of $D_{p}$ will get smaller, forcing the search to take smaller steps and "zoom in" on the PAE boundary.

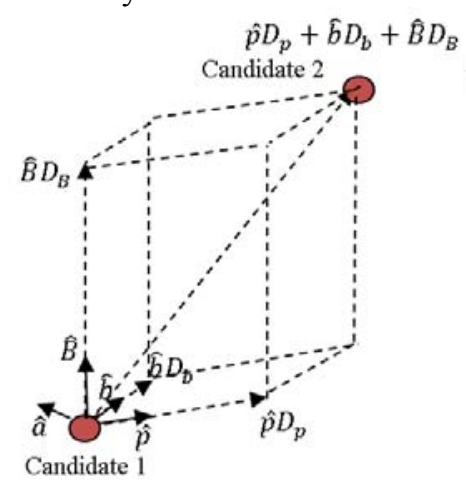

(a)

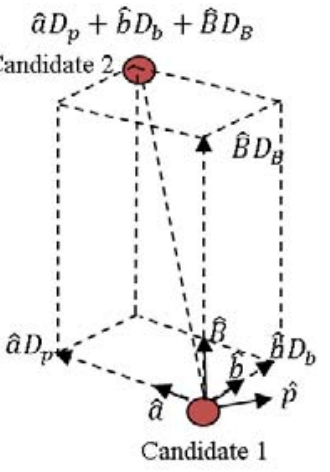

(b)
Fig. 4. (a) Calculation of the next candidate point in the case where the PAE is not acceptable at Candidate 1, and (b) calculation of the next candidate point in the case where the PAE is acceptable at Candidate 1

The component in the direction of the bisector between optimum PAE and ACPR directions, $D_{b}$, as prescribed by equation (5), is designed to take the search toward the Pareto optimum locus. The Pareto optimum locus consists of the constrained optimum solutions for minimum ACPR under different PAE constraints, and extends from the ACPR optimum to the PAE optimum as the PAE constraint is varied. On the Pareto optimum locus, the unit vectors $\hat{p}$ and $\hat{a}$ are collinear [27], and therefore the angle of $\theta$ on this Pareto optimum locus is 90 degrees. The solution we desire lies on the Pareto optimum locus: this means that the value of $\theta_{\text {target }}$ in equation (5) is 90 degrees. As the search unfolds, it should approach the Pareto optimum locus at the limiting value of PAE through the summation of (4) and (5) in the search vector. Both of these equations use $D_{s} / 2$ as the base search distance that is modified by the estimator of how close the search is to either the PAE limitation or the Pareto optimum locus. The maximum magnitude of the search vector's projection into the $\Gamma_{L}$ plane is $D_{s}$.

Equation (6) gives the component of the search vector in the vertical (bandwidth $B$ ) direction within the Smith Tube. The components in the $\hat{a}$ and $\hat{b}$ directions steer the solution toward the constrained optimum ACPR (within PAE requirements) in the Smith Chart plane. The vertical component of the search vector, $\hat{B} D_{B}$, is designed to take the search vertically to the edge of the ACPR acceptable region. Because the PAE acceptable region is expected to be nearly independent of bandwidth, the search vector is designed to place the candidate within the ACPR acceptable region, then ascend vertically in the Smith Tube until it stops upon reaching the ACPR limit. Equation (6) contains a ratio that describes the relative ACPR change needed to reach the limit 
with respect to the ACPR change accomplished in a vertical neighboring-point measurement. This component will force the search vector to have an upward direction if (6) is positive and a downward direction if (6) is negative.

\section{Measurement Search Results}

The accuracy and efficiency of the search algorithm are demonstrated through measurement results. Figure 5 shows the laboratory measurement setup. MATLAB is used to run the algorithms and program the input waveform into the Agilent Technologies N5182 MXG signal generator. The tuning of the load reflection coefficient is performed using a Maury Microwave MT982B mechanical tuner under control of the Maury Automated Tuner System software. Power measurements for calculation of the PAE are taken using an Agilent AT N911A power meter and N1921A power sensor. An Agilent E4407B $9 \mathrm{kHz}$ to $26.5 \mathrm{GHz}$ spectrum analyzer are used for ACPR measurements. A Skyworks packaged amplifier was used as the test device for this paper. The input power to the device was $2 \mathrm{dBm}$ for all measurements shown. This power level results in approximately $2 \mathrm{~dB}$ compression.

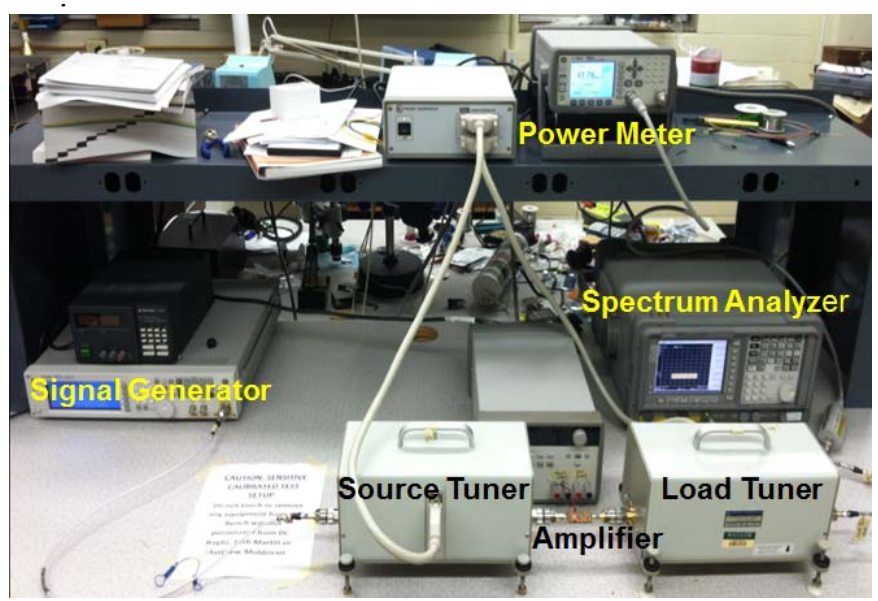

Fig. 5. Laboratory measurement setup

The search was performed for multiple starting combinations of $\Gamma_{L}$ and $B$. The trajectories of the searches are shown in Figures 6 through 12 for the different starting points. For these searches, the combination of $\Gamma_{L}$ and $B$ resulting in the highest bandwidth while providing ACPR $\leq$ $-27.5 \mathrm{dBc}$ and $\mathrm{PAE} \geq 7 \%$ is sought. In each case, the starting $\Gamma_{L}$ and $B$, as well as the bandwidth search range, are specified in the figure caption. As an example, Figure 6 plots the search trajectory for starting $\Gamma_{L}=0.44 /-20^{\circ}$ and a specified bandwidth search range of $15 \mathrm{MHz}$ to $20 \mathrm{MHz}$, placing the starting bandwidth at the center of this range $(17.5 \mathrm{MHz})$. Figure 6(a) shows a three-dimensional view of the measured triangulation algorithm Smith Tube search trajectory in threedimensions, and Figure 6(b) shows projection of the search's progress in the $\Gamma_{L}$ plane. The optimum point in the Smith Tube for this search was found to be $\Gamma_{L}=0.573 /-18.66^{\circ}, B=$ 16.635 MHz. This combination resulted in ACPR $=-27.58$ $\mathrm{dBc}$ and $\mathrm{PAE}=7.05 \%$, and was reached with a total of 21 measurements. Table I shows the end $B$ and $\Gamma_{L}$ values, as well as the final values of ACPR and PAE and number of measurements for all of the searches shown in Figures 6 through 12.

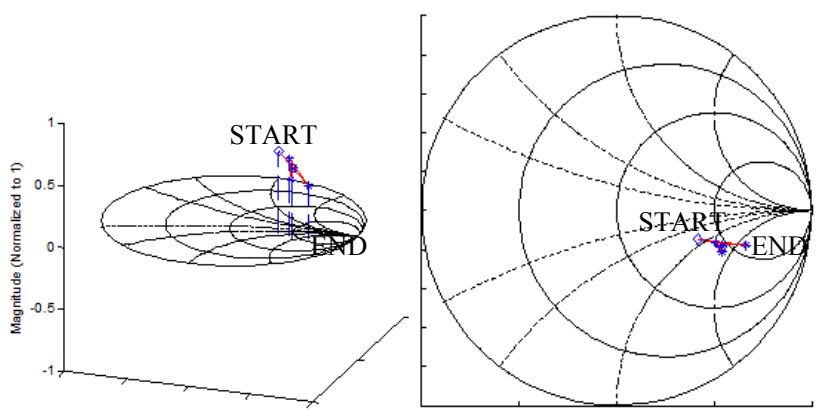

(a)

(b)

Fig. 6. (a) Three-dimensional view of the measured triangulation algorithm Smith Tube search trajectory for a search starting from $\Gamma_{L}=0.44 /-20^{\circ}$ and $B=17.5 \mathrm{MHz}$ (the bandwidth search range of 15 $\mathrm{MHz}$ to $20 \mathrm{MHz}$ is normalized to operate between -1 and 1 , where the starting point, the range center, is assigned to be zero), and (b) horizontal projection of the search

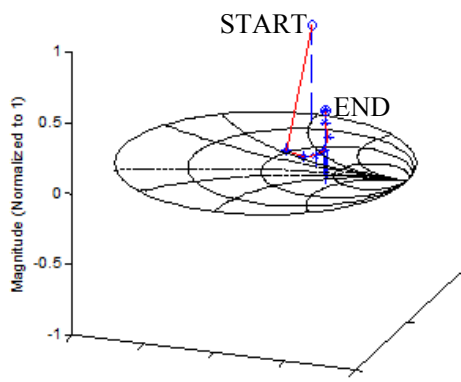

(a)

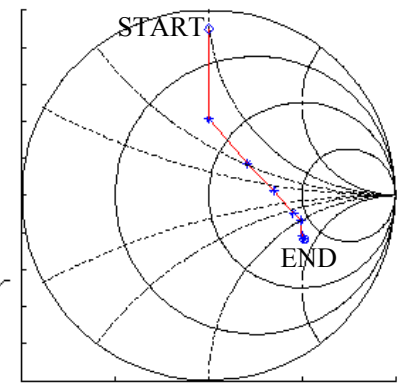

(b)
Fig. 7. (a) Three-dimensional view of the measured triangulation algorithm Smith Tube search trajectory for a search starting from $\Gamma_{L}=0.9 / 90^{\circ}$ and $B=17.5 \mathrm{MHz}$ (the bandwidth search range of 15 $\mathrm{MHz}$ to $20 \mathrm{MHz}$ is normalized to operate between -1 and 1, where the starting point, the range center, is assigned to be zero), and (b) horizontal projection of the search

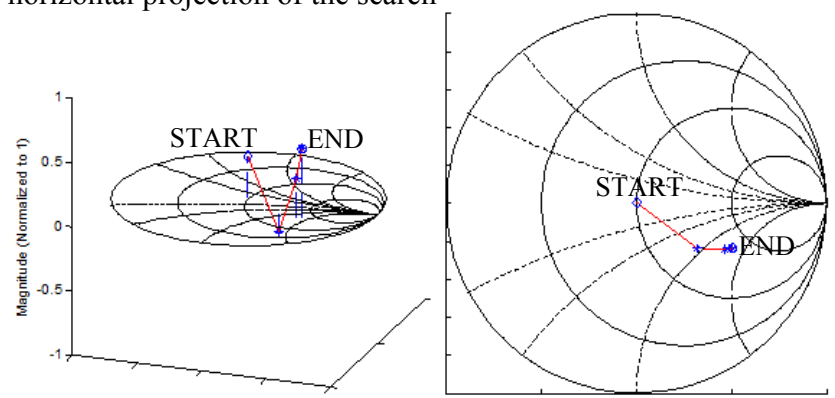

(a)

(b)

Fig. 8. (a) Three-dimensional view of the measured triangulation algorithm Smith Tube search trajectory for a search starting from $\Gamma_{L}=0$ and $B=15 \mathrm{MHz}$ (the bandwidth search range of $10 \mathrm{MHz}$ to $20 \mathrm{MHz}$ is normalized to operate between -1 and 1 , where the starting point, the range center, is assigned to be zero), and (b) horizontal projection of the search

Figure 6 shows that for a starting $\Gamma_{L}=0.44 /-20^{\circ}$ and bandwidth search range from $15 \mathrm{MHz}$ to $20 \mathrm{MHz}$, the search 
slowly decreases the value of $B$ and moves the $\Gamma_{L}$ toward the endpoint of the search. The initial value of $B, 17.5 \mathrm{MHz}$, is higher than the final bandwidth of $16.635 \mathrm{MHz}$. Figure 7 shows the results of the search starting from $\Gamma_{L}=0.90 / 90^{\circ}$ and bandwidth search range from $15 \mathrm{MHz}$ to $20 \mathrm{MHz}$. The search first descends rapidly; in fact, it seems (on initial instinct) to "overreact". This is because the change in ACPR with slight vertical displacement is formidable enough to cause the bandwidth to be changed significantly.

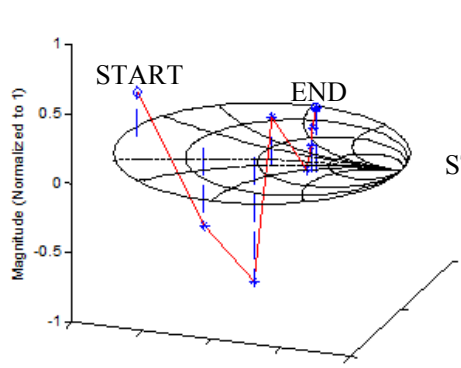

(a)

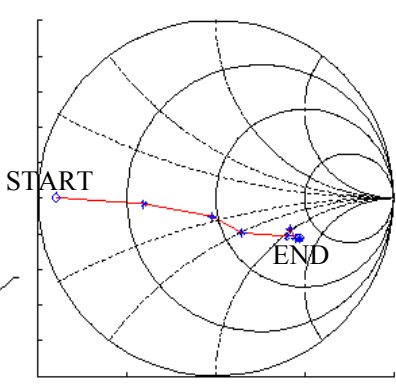

(b)
Fig. 9. (a) Three-dimensional view of the measured triangulation algorithm Smith Tube search trajectory for a search starting from $\Gamma_{L}=0.9 / 180^{\circ}$ and $B=15 \mathrm{MHz}$ (the bandwidth search range of 10 $\mathrm{MHz}$ to $20 \mathrm{MHz}$ is normalized to operate between -1 and 1 , where the starting point, the range center, is assigned to be zero), and (b) horizontal projection of the search

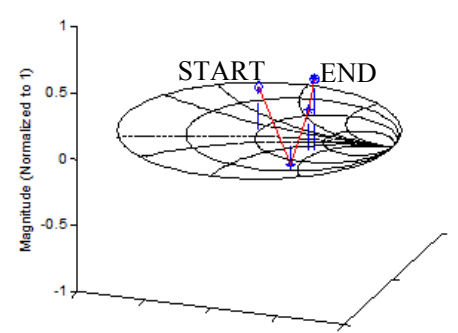

(a)

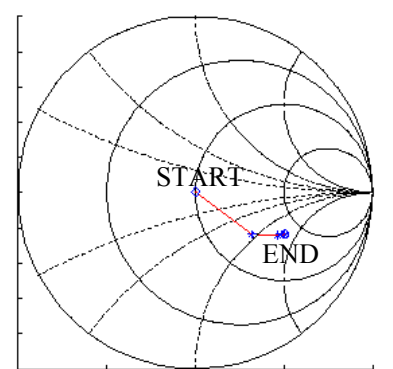

(b)
Fig. 10. (a) Three-dimensional view of the measured triangulation algorithm Smith Tube search trajectory for a search starting from $\Gamma_{L}=0$ and $B=15 \mathrm{MHz}$ (the bandwidth search range of $10 \mathrm{MHz}$ to $20 \mathrm{MHz}$ is normalized to operate between -1 and 1 , where the starting point, the range center, is assigned to be zero), and (b) horizontal projection of the search

In any case where the ACPR at a candidate is above the acceptable limit, the subsequent candidate will be below its predecessor in the Smith Tube. In addition to Fig. 6, this is the case in the searches displayed in Figures 7, 8, 9, 10, and 12. In some cases, the starting value of $B$ is actually lower than the final bandwidth resulting from the optimization, but the ACPR does not meet requirements due to the fact that $\Gamma_{L}$ is significantly displaced from the ACPR optimum on the Smith Chart. Based on equation (6), the search only proceeds upward when the ACPR at a candidate is within the acceptable ACPR limit. Thus the search proceeds downward until an acceptable value of ACPR is obtained, then it will proceed upward while the ACPR remains acceptable. As the search proceeds downward, the value of $\Gamma_{L}$ is also optimized; both of these components cause improvement in ACPR. The search then can proceed upward until the ACPR leaves the compliance region, or until the PAE and ACPR limits are reached and the constrained optimum is determined. The value of $D_{s}$ is divided by 2 if the candidate, after having entered the intersection of the PAE and ACPR acceptable regions, attempts to leave the intersection of the PAE and ACPR acceptable regions. This causes the search distance to be lowered further as the optimum is approached.

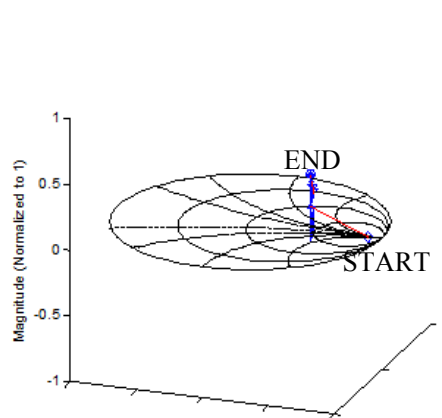

(a)

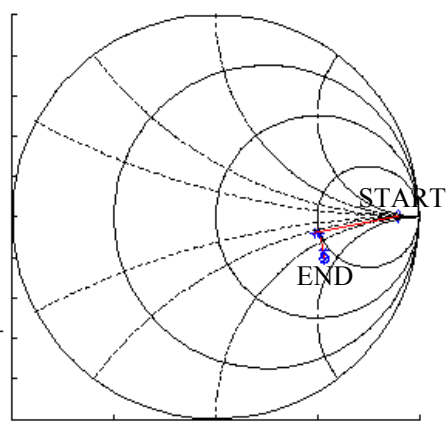

(b)
Fig. 11. (a) Three-dimensional view of the measured triangulation algorithm Smith Tube search trajectory for a search starting from $\Gamma_{L}=0.9 / 0^{\circ}$ and $B=12.5 \mathrm{MHz}$ (the bandwidth search range of 5 $\mathrm{MHz}$ to $20 \mathrm{MHz}$ is normalized to operate between -1 and 1 , where the starting point, the range center, is assigned to be zero), and (b) horizontal projection of the search

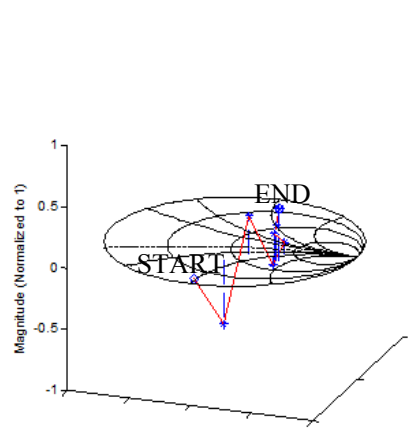

(a)

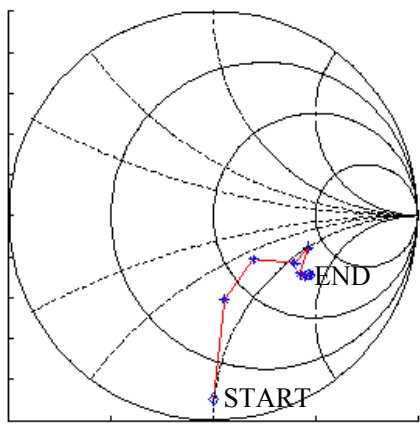

(b)
Fig. 12. (a) Three-dimensional view of the measured triangulation algorithm Smith Tube search trajectory for a search starting from $\Gamma_{L}=0.9 /-90^{\circ}$ and $B=12.5 \mathrm{MHz}$ (the bandwidth search range of 5 $\mathrm{MHz}$ to $20 \mathrm{MHz}$ is normalized to operate between -1 and 1 , where the starting point, the range center, is assigned to be zero), and (b) horizontal projection of the search

\section{TABle I: Alorithm Measurement Search Results For Multiple STARTING CONDITIONS}

\begin{tabular}{|c|c|c|c|c|c|c|}
\hline $\begin{array}{c}\text { Start } \\
B \\
(\mathrm{MHz})\end{array}$ & $\begin{array}{c}\text { Start } \\
\Gamma_{L}\end{array}$ & $\begin{array}{c}\text { End } \\
B \\
(\mathrm{MHz})\end{array}$ & End $\Gamma_{L}$ & $\begin{array}{c}\text { End } \\
\text { ACPR } \\
(\mathrm{dBc})\end{array}$ & $\begin{array}{c}\text { End } \\
\text { PAE } \\
(\%)\end{array}$ & $\begin{array}{c}\# \\
\text { Meas. }\end{array}$ \\
\hline 17.5 & $0.44 /-20^{\circ}$ & 16.635 & $0.573 /-18.66^{\circ}$ & -27.58 & 7.05 & 21 \\
\hline 17.5 & $0.90 \underline{/ 90^{\circ}}$ & 16.377 & $0.562 /-24.82^{\circ}$ & -27.63 & 7.27 & 34 \\
\hline 15 & 0 & 16.498 & $0.557 /-25.26^{\circ}$ & -27.50 & 7.25 & 18 \\
\hline 15 & $0.9 / 180^{\circ}$ & 15.959 & $0.519 /-25.38^{\circ}$ & -27.67 & 7.48 & 33 \\
\hline 12.5 & $0.9 / 0^{\circ}$ & 16.258 & $0.576 /-21.11^{\circ}$ & -27.54 & 7.29 & 22 \\
\hline 12.5 & $0.9 \underline{/-90^{\circ}}$ & 15.732 & $0.555 /-31.42^{\circ}$ & -28.11 & 7.40 & 34 \\
\hline
\end{tabular}


The results of Table I show good correspondence between the end $B$ and $\Gamma_{L}$ values across the different starting conditions. In addition, all of the measurements show PAE and ACPR values that are near their limits $(7$ percent and $-27.5 \mathrm{dBc}$, respectively), yet within compliance. For the cases shown, the algorithm demonstrates a good estimation of the constrained optimum $\left(\Gamma_{L}, B\right)$ combination with between 18 and 34 measurements. While estimates of actual optimization time in real radar systems for this number of measurements have yet to be developed, the reduction in the number of measurements is significant compared to the "brute-force" exhaustive search method of performing loadpull measurements at multiple bandwidths [2].

\section{CONCLUSIONS}

This paper demonstrates a new measurement-based algorithm that allows fast load-impedance and waveform optimization to maximize bandwidth while meeting poweradded efficiency and adjacent-channel power ratio requirements. Good convergence of this algorithm is demonstrated from multiple starting combinations of load reflection coefficient and bandwidth. This algorithm is expected to provide fast real-time, reconfiguration of power amplifier circuitry and input waveform for future radar transmitters. Observation of the algorithm for multiple starting points shows that the search endeavors to reach the acceptable ACPR region quickly, indicating that the device will maintain spectrally compliant (or near-compliant) operation for a considerable portion of the testing. This work uses the Smith Tube, a new tool for joint, simultaneous optimization of radar circuit and waveform parameters.

\section{ACKNOWLEDGMENTS}

This work has been funded under a grant from the National Science Foundation (Award Number ECCS-1343316). The authors are appreciative to Dr. Eric Mokole, formerly of the U.S. Naval Research Laboratory, for the idea of jointly optimizing the circuit and waveform in power amplifiers.

\section{REFERENCES}

[1] M. Fellows, C. Baylis, J. Martin, L. Cohen, and R.J. Marks II, "Direct Algorithm for the Pareto Load-Pull Optimisation of Power-Added Efficiency and Adjacent-Channel Power Ratio," Accepted October 2013 for publication in IET Radar, Sonar \& Navigation.

[2] M. Fellows, M. Flachsbart, J. Barlow, C. Baylis, L. Cohen, and R.J. Marks II, "The Smith Tube: Selection of Radar Chirp Waveform Bandwidth and Power Amplifier Load Impedance Using MultipleBandwidth Load-Pull Measurements," 2014 IEEE Wireless and Microwave Technology Conference (WAMICON 2014), Tampa, Florida, June 2014.

[3] M. Fellows, M. Flachsbart, J. Barlow, J. Barkate, C. Baylis, L. Cohen, and R.J. Marks II, "Optimization of Power Amplifier Load Impedance and Waveform Bandwidth for Real-Time Reconfigurable Radar," submitted to IEEE Transactions on Aerospace and Electronic Systems, May 2014.

[4] J. Sevic, K. Burger, and M. Steer, "A Novel Envelope-Termination Load-Pull Method for ACPR Optimization of RF/Microwave Power Amplifiers," 1998 IEEE MTT-S International Microwave Symposium Digest, June 1998, Vol. 2, pp. 723-726.

[5] J. Sevic, "Large Signal Automated Load-Pull Characterization of
Adjacent-Channel Power Ratio for Digital Wireless Communication Systems," 1996 IEEE MTT-S International Microwave Symposium Digest, June 1996, pp. 763-766.

[6] Q. Wu, H. Xiao, and F. Li, "Linear Power Amplifier Design for CDMA Signals: A Spectrum Analysis Approach," Microwave Journal, 1998.

[7] F.N. Sechi, "Design Procedure for High-Efficiency Linear Microwave Power Amplifiers," IEEE Transactions on Microwave Theory and Techniques, Vol. 28, Pt. 1, November 1980, pp. 1157-1163.

[8] Y. Sun, "Evolutionary Tuning Method for Automatic Impedance Matching in Communication Systems," Proceedings of the 1998 IEEE International Conference on Electronics, Circuits, and Systems, Vol. 3, pp. 73-77.

[9] Y. Sun and W.K. Lau, "Antenna Impedance Matching Using Genetic Algorithms," Proceedings of the IEE Conference on Antennas and Propagation, York, United Kingdom, August 1999, pp. 31-36.

[10] E. Arroyo-Huerta, A. Diaz-Mendez, J. Ramirez Cortes, and J. Garcia, "An Adaptive Impedance Matching Approach Based on Fuzzy Control," $52^{\text {nd }}$ IEEE International Midwest Symposium on Circuits and Systems, August 2009, pp. 889-892.

[11] J. Hemminger, "Antenna Impedance Matching with Neural Networks," International Journal of Nerual Systems (IJNS), Vol. 15, No. 5, pp. 357-361, 2005.

[12] A. Mushi, D. Johns, and A. Sedra, "Adaptive Impedance Matching," proceedings of the IEEE International Symposium on Circuits and Systems, 1994 (ISCAS '94), Vol. 2, pp. 69-72.

[13] Y. Sun, J. Moritz, and X. Zhu, "Adaptive Impedance Matching and Antenna Tuning for Green Software-Defined and Cognitive Radio," $54^{\text {th }}$ IEEE International Midwest Symposium on Circuits and Systems, 2011, pp. 1-4.

[14] N. Kingsley and J.R. Guerci, "Adaptive Amplifier Module Technique to Support Cognitive RF Architectures," 2014 IEEE Radar Conference, Cincinnati, Ohio, May 2014, pp. 1329-1332.

[15] R. Chen and B. Cantrell, "Highly Bandlimited Radar Signals," Proceedings of the 2002 IEEE Radar Conference, pp. 220-226.

[16] S. Blunt, M. Cook, E. Perrins, and J. de Graaf, "CPM-Based Radar waveforms for Efficiently Bandlimiting a Transmitted Spectrum," 2009 IEEE Radar Conference, pp. 1-6.

[17] M. Cook, M.S. Thesis: CPM-Based Radar Waveforms for Efficiently Bandlimiting a Transmitted Spectrum, University of Kansas, April 2010.

[18] M. Moldovan, C. Baylis, R.J. Marks II, M. Wicks, and J. Martin, "Chirp Optimization Using Piecewise Linear Approach," International Waveform Diversity and Design Conference, Kauai, Hawaii, January 2012.

[19] M. Fellows, C. Baylis, L. Cohen, and R.J. Marks II, "Radar Waveform Optimization to Minimize Spectral Spreading and Achieve Target Detection," Texas Symposium on Wireless and Microwave Circuits and Systems, Waco, Texas, April 2013.

[20] L. Patton, On the Satisfaction of Modulus and Ambiguity Function Constraints in Radar Waveform Optimization for Detection, Doctoral Disseration, Wright State University, 2007.

[21] J. Holtzman and J. Thorp, "Optimum Signals for Radar," IEEE Transactions on Aerospace and Electronic Systems, Vol. 5, No. 6, pp. 898-905, November 1969

[22] K. Wong and W.-K. Chung, "Pulse-Diverse Radar/Sonar FSK-PSK Waveform Design to Emphasize/De-Emphasize Designated DopplerDelay Sectors," IEEE Radar Conference, Alexandria, Virginia, May 200 , pp. 745-749.

[23] S. Sussman, "Least-Square Synthesis of Radar Ambiguity Functions," IRE Transactions on Information Theory, Vol. 8, No. 3, pp. 246-254, April 1962.

[24] J. Jakabosky, S.D. Blunt, M.R. Cook, J. Stiles, and S. Seguin, "Transmitter-in-the-Loop Optimization of Physical Radar Emissions," IEEE Radar Conference, May 2012, Atlanta, Georgia.

[25] S. Haykin, "Cognitive Radar: A Way of the Future," IEEE Signal Processing Magazine, Vol. 23, No. 1, pp. 30-40.

[26] J.R. Guerci, Cognitive Radio: The Knowledge-Aided Fully Adaptive Approach, Artech House, 2010.

[27] J. Martin, C. Baylis, L. Cohen, J. de Graaf, and R.J. Marks II, “A PeakSearch Algorithm for Load-Pull Optimization of Power-Added Efficiency and Adjacent-Channel Power Ratio," IEEE Transactions on Microwave Theory and Techniques, Vol. 62, No. 8, August 2014, pp. 1772-1783. 